\title{
Continuing education for healthcare professionals: time to prove its worth
}

\author{
*Monica Fletcher \\ Chief Executive, Education for Health, 10 Church Street, Warwick, CV34 4AB, UK
}

Received 28th April 2007; accepted 6th May 2007

\begin{abstract}
Summary
With escalating financial pressures being placed upon health services, it becomes increasingly hard to justify spending on education and training. At the same time, however, primary care practitioners are being expected to take on new roles in the management of long term conditions which were once the domain of their secondary care colleagues. This Discussion paper highlights the importance of expanding the evidence base for healthcare professional training - particularly in terms of its positive effects on patient care - and emphasises how important it is that those responsible for commissioning training continue to see the importance of doing so. It cites a randomised controlled trial of education in the primary care management of rhinitis as an example of the way in which healthcare professional education can improve patients' quality of life. Without continued investment in healthcare professionals' education, the vision of high quality, guideline-driven, evidence-based health care will never come to fruition.

(c) 2007 General Practice Airways Group. All rights reserved.

M Fletcher. Prim Care Resp J 2007; 16(3): 188-190.

doi:10.3132/pcrj.2007.00041
\end{abstract}

Keywords education, training, primary care, allergy, quality of life

\section{Introduction}

Health care systems around the globe, whether they are government-funded as in the UK, or insurance-funded as in the USA, or have a mix of public and private financing, are facing increasing financial pressures. As scientific and technological advances are made, more is known about the pathogenesis, prevention and treatment of both acute and chronic illnesses. The expectations of individual patients and also of society as a whole - are increasing, and as life expectancy rises, ${ }^{1}$ resources will become ever more stretched.

People with chronic respiratory and allergic conditions are just one group of patients fighting to get some of the limited resources. The majority of these patients do not require 'high tech' and expensive care from hospitals, but they do need access to high quality primary care services delivered by appropriately-trained primary health care professionals working as part of a team.

During the last year, the financial difficulties of the UK National Health Service (NHS) have become acutely obvious to both the general public and those working in the service.
Newly-created primary care organisations (PCOs) and Strategic Health Authorities have grappled with financial deficits and structural reorganisations. Many have chosen to take a short-term view and have raided education budgets to offset these deficits.

It is of no surprise, therefore, that within this difficult climate, maintenance of funding streams for training health professionals has been given a low priority. However, this is short sighted, may well prove detrimental to patient care, and is likely to affect job satisfaction and fuel the crisis of retention of clinical staff. This Discussion paper highlights some of the issues involved, and in particular documents recent evidence which confirms that training of healthcare professionals can alter patient outcomes.

\section{The importance and costs of continuing professional education}

Unfortunately, continuing professional development (CPD) and continued medical education (CME) are costly in terms of organisational resources such as time and money as well as the personal effort involved. 
At a time when UK NHS health policy purports to support a drive towards an ever-expanded Primary Care-led NHS, those working in primary care appreciate that ensuring that the workforce is clinically up to date and well educated is of paramount importance. Primary care has taken on many of the roles and responsibilities that were once the domain of their secondary care colleagues: indeed, roles have blurred considerably. However, new roles require new knowledge, skills and competencies, and that is why training must not be omitted from any health service planner's agenda.

In contrast to the restrictions imposed by budgetary constraints, increasing consumerism and patient empowerment as well as an increased emphasis on efficiency and effectiveness have led to a drive to raise clinical standards and to reduce inequalities and variance in care. Nevertheless, we live in a world that looks for a 'return on investment.' Anecdotal claims that education for healthcare professionals makes a difference to care, without evidence to back those claims up, will no longer stand scrutiny in the brave new world of 'value for money'.

A review of the literature reveals that there is little high quality evidence on the impact of healthcare professional education on the quality of services or on patient outcomes. Unfortunately, if there is limited evidence that any change in clinical practice results in improvements to patient care, health service funders will continue to be in the position to argue that spending on training has a lower priority than other Government-driven targets. However, a lack of evidence does not mean that education has no benefit, just that the evidence is not there to prove it. We urgently need educational researchers to build the evidence base, since the rationale for continuing education is based on the assumption that education and training will improve patient care.

\section{Evidence in favour of continuing professional education}

There is evidence that education improves the confidence and competence of practitioners, ${ }^{2}$ and changes to practice as a result of education have also been documented. ${ }^{3-5}$ However, linking professional education directly to patient outcomes is difficult to demonstrate.

Since Education for Health is a training charity, we felt that it was vital to test the assumption that educational interventions do indeed positively affect patient outcomes. We chose to study the effect of a training intervention on patients living with rhinitis because it is one of the most common chronic conditions, affecting up to $30 \%$ of the UK population. ${ }^{6}$ Rhinitis symptoms frequently result in significant morbidity and may adversely affect concentration, ${ }^{7}$ reduce productivity, and impair learning ability in children and adolescents. ${ }^{8}$ In addition, patients experience impairment in quality of life through systemic symptoms, sleep disturbance, practical problems, activity limitations and emotional problems. ${ }^{7}$ The costs associated with lost work days due to rhinitis are also significant; a recent American study showed that allergic rhinitis resulted in approximately 811,000 missed work days, 824,000 missed school days and 4,230,000 reduced activity days. ${ }^{9}$

The majority of patients with rhinitis can be managed successfully in primary care. A document aiming to provide a blueprint for better patient care and published by the Royal College of Physicians ${ }^{6}$ recommends that 'the front line for allergy management must be within primary care', under clinical leadership from specialist centres. The report recommends improved access to postgraduate training as an essential prerequisite to improving allergy practice in primary care.

Therefore, we recently conducted a multi-centre community-based parallel group randomised trial of an educational intervention (the Education for Health training course) versus normal care, and assessed its impact on the care of patients with rhinitis by measuring quality of life outcomes. ${ }^{10}$ Twenty general practitioners (GPs) and practice nurses with no previous allergy training were recruited from 12 UK general practices. One hundred and fifty-seven patients received the intervention in accordance with the protocol (patients per protocol analysis, PPA). The training consisted of Education for Health's Allergy Course and was delivered over a six-month period using a combination of a distance-learning package and three days of face-to-face instruction. The primary outcome measure was the change from baseline in the validated Rhino-conjunctivitis Quality of Life Questionnaire (RQLQ) ${ }^{11}$ between the two groups at 13 months after randomisation i.e. six months after completion of the intervention. Effects on professional competence and confidence were measured (on completion of the training intervention and six months later). An overall evaluation of satisfaction with the allergy training module was completed.

The study showed significant improvements in RQLQ from baseline in the intervention group (baseline 2.03 vs. six months 1.8), but not in the control group (1.8 vs. 1.7; intervention vs. control, $p=0.08)$. In the PPA, RQLQ scores improved significantly in the intervention group (2.0 vs. 1.8), but not in the control group (1.8 vs.1.8; intervention vs. control, $p=0.05)$. The number of patients needed to treat (NNT) for one patient to have a clinically meaningful improvement (i.e. $\geq 0.5$ ) was nine. Healthcare professional self-rated confidence and behaviours improved across a range of clinical indicators and over three-quarters were of the opinion that they had acquired new knowledge and skills.

Therefore, in this recently-published study, standardised allergy training was well evaluated by healthcare 
professionals and resulted in improvements in healthcare professionals' perceived confidence in managing allergic conditions. What is of greater significance is that there were improvements in disease-specific health-related quality of life in patients with perennial rhinitis.

\section{Conclusion}

These findings make a substantial contribution to the scientific literature and they highlight the importance of continued investment in professional education. In today's climate of financial instability, and in a culture of evidencebased practice, it is important to show the clear benefits of healthcare professional education for both patients and health professionals. The diminishing priority given to funding the education of the primary care workforce in the UK cannot be allowed to continue. Without investment in healthcare professionals' education, the vision of high quality, guidelinedriven, evidence-based health care will never come to fruition.

\section{Conflict of interest declaration}

The author is Chief Executive of Education for Health. Education for Health (previously known as the National Respiratory Training Centre) is an independent, not-for-profit charitable organisation which delivers Open University-accredited diploma and degree level modules in allergic, respiratory and cardiovascular disease, with the aim of improving patient care. Over the last 20 years the charity has trained over 40,000 health professionals in the management of long-term conditions, including allergic disease.

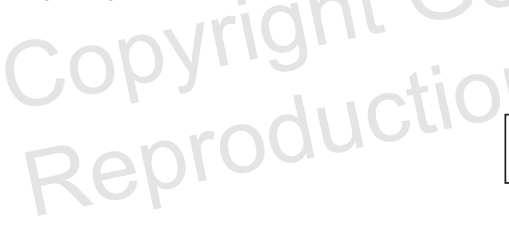

\section{References}

1. World Health Organization. The challenge of chronic conditions. Preparing a Health Care Workforce for the 21st century. BMJ 2005;330:637-9.

2. Coomber J, Lester M, Yeatts K, Fletcher M, Walker S. Allied Health Professional Education and Enhancement of the Practitioner's Role. Eur Resp J 2006. Proceedings of the European Respiratory Society, p835

3. Coomber J, Yeatts K, Lester M, Fletcher M, Walker S. Improving Asthma Diagnosis through Health Practitioner Education. Am J Respir Crit Care Med 2006;B23:A292.

4. Garrad J, Choudary V, Groom H, Dieperink, Willenbring ML, Durfee JM, Ho SB. Organisational Change in Management of Hepatitis C: Evaluation of a CME program J Contin Educ Health Prof 2006; 26(2):145-60.

5. Lougheed D, Moosa D, Finlayson S, Hopman WM, Quinn M, Szpiro K, Reisman J. Impacts of a provincial asthma guidelines continuing medical education project: The Ontario Asthma Plan of Action's Provider Education in Asthma Care Project. Can Respir J 2007;14(2):111-17.

6. Royal College of Physicians. Allergy: the unmet need. A blueprint for better patient care, Royal College of Physicians, London; 2003.

7. Juniper EF, Guyatt GH. Development and testing of a new measure of health status for clinical trials in rhinoconjunctivitis. Clin Exp Allergy 1990;21:77-83.

8. Vuurman EPF, van Veggel LMA, Uiterwijk MMC, Leutner D, O'Hanlon JF. Seasonal allergic rhinitis and anti-histamine effects on childrens' learning. Ann Allergy 1993;71:121-6.

9. Malone DC, Lawson KA, Smith DH, Arrighi HM, Battista C. A cost of illness study of allergic rhinitis in the United States. J Allergy Clin Immunol 1997; 99:22-7.

10. Sheikh A, Khan-Wasti S, Price D, Smeeth L, Fletcher M, Walker S. Standardized training for healthcare professionals and its impact on patients with perennial rhinitis: a multi-centre randomized controlled trial. Clin Exp Allergy 2007; 37(1):90-9.

11. Juniper EF, Thompson AK, Ferrie PJ, Roberts JN. Development and validation of the Mini-rhinoconjunctivitis Quality of Life Questionnaire. Clin Exp Allergy 2000;30:132-40.

Available online at http://www.thepcrj.org 